\title{
The Relationship Between Different Care Units Nurses and Fall Prevention Knowledge in Acute Care Hospitals in China Based on Lasso Regression Model
}

\author{
Lin Wang ${ }^{1}$, Li Zhang ${ }^{2}$, Weiju Chen ${ }^{3}$, Zhiwei Mou ${ }^{4, *}$ \\ ${ }^{1}$ Transitional Care Department, the First Affiliated Hospital of Jinan University, Guangzhou, China \\ ${ }^{2}$ Department of First Class Ward, the First Affiliated Hospital of Jinan University, Guangzhou, China \\ ${ }^{3}$ School of Nursing, Jinan University, Guangzhou, China \\ ${ }^{4}$ Department of Rehabilitation Medicine, the First Affiliated Hospital of Jinan University, Guangzhou, China \\ Email address: \\ wanglin18903078509 (Lin Wang), mouzhiwei@jnu.edu.cn (Zhiwei Mou) \\ ${ }^{*}$ Corresponding author
}

\section{To cite this article:}

Lin Wang, Li Zhang, Weiju Chen, Zhiwei Mou. The Relationship Between Different Care Units Nurses and Fall Prevention Knowledge in Acute Care Hospitals in China Based on Lasso Regression Model. American Journal of Nursing Science. Vol. 9, No. 3, 2020 , pp. 175-180. doi: 10.11648/j.ajns.20200903.28

Received: April 30, 2020; Accepted: May 22, 2020; Published: May 29, 2020

\begin{abstract}
Objective: To explore the difference of fall prevention knowledge level of nurses working on special care units and general care units in acute hospitals. Methods: Nurses in special care units and general care units from two acute care hospitals were investigated about fall prevention through a cross-sectional survey. The collected data was analyzed by using Lasso Regression Model. Results: Total of 1442 valid questionnaires were conducted including 1028 questionnaires in general care units and 414 questionnaires in special care units respectively. Through analysis by Lasso Regression Model, seven of 17 fall prevention knowledge items were statistically different in nurses working on general care units and special care units, including the definition of a fall $(\mathrm{P}=0.0001)$, fall prevention assessment tool currently used $(\mathrm{p}=0.0053)$, assessment of patient's balance and activity ( $\mathrm{p}=0.0020)$, diseases that easily cause patients to fall $(\mathrm{p}=0.0021)$, assessment of patient's foot problems $(\mathrm{p}=0.0068)$, assessments of the patient's clothing and footwear suitability $(\mathrm{p}=0.0011)$, reporting process of falls $(\mathrm{p}<0.0001)$. Conclusion: This study showed that nurses in special care units have higher level theoretical knowledge of fall prevention than nurses in general care units, while having lower level practical knowledge of fall prevention. It suggested that Quality \& Patient Safety Department in medical institution should make new plans to prevent patients from falling and ensure patient safety.
\end{abstract}

Keywords: Least Absolute Shrinkage and Selection Operator (Lasso), Knowledge of Fall Prevention, Nurses in Different Care Units

\section{Introduction}

Fall is a serious international public health issue affecting individuals at any age, which is the second leading cause of accidental or unintentional injury deaths worldwide [1]. There are estimated 646,000 individuals dying from falls globally of which over $80 \%$ are in low- and middle-income countries every year [1]. In China, with the rapid aging of the population, fall have become the leading cause of injury for the aged over 65 [2]. The consequences of falls are manifold, including physical, psychological, social, economic, and sometimes even fatal in which one third of falls in hospital results in harm of varying degrees $[3,4]$.

Fall prevention in clinical practice is a complex undertaking that requires the collaboration of multiple disciplines which includes nursing, physicians, environmental services, information technology, patient advocacy, pharmacy, physical and occupational therapy. Although falls prevention is a multidisciplinary task, nurses are the leaders in this initiative. In clinical practice, nurses are in the frontline and are mainly responsible for the assessment of the risk of falls as well as health education of the patients [5]. It requires nurses to be equipped with knowledge and skills of fall prevention to 
perform their roles effectively.

The majority of the study found focus on the incidence of falls, the risk factors of falls, and fall prevention in patients, which are mainly distributed in the general care units of medical, surgical, obstetrics and pediatrics [6-9]. In the recent years, a few researches have reported the incidence of falls and fall prevention in special care units such as emergency department, intensive care unit and operating room [10-12]. As an important role in clinical practice, nurses' knowledge directly relates to their attitude towards implementing fall prevention intervention measures. There are some studies investigating about the knowledge of fall prevention of nurses in the hospitals $[13,14]$, while that of nurses in special care units is rare. Therefore, it is necessary to study about the fall prevention knowledge level of nurses in special care units. The aim of this study was to use the least absolute shrinkage and selection operator (Lasso) Logistic regression model to analyze the difference of fall prevention knowledge level of nurses in special care units and general care units so that to provide reference for Quality \& Patient Safety Department in medical institution and to develop specific nurses' training programs about fall prevention.

\section{Methods}

\subsection{Participants and Recruitment}

There were 1880 nurses in 2 acute care hospitals of Guangzhou that participants were recruited from a total of 1600 nurses according to inclusion and exclusion criteria. Inclusion criteria: Over 18 years old; licensed nurses providing direct patient care from general care units including medical, surgical, obstetrics \& gynecology, pediatric, while special care units including emergency department, intensive care unit, operation room. Exclusion criteria: Nurse administrators or licensed nurses working in nonclinical roles. The Ethical approval was obtained from Institutional Review Board of both hospitals. Recruitment took place from October $20^{\text {th }}$ to November $20^{\text {th }}, 2018$.

\subsection{Study Design}

The study used a cross-sectional survey which included fall prevention knowledge of participants and their demographic information such as years of experience, education level and working unit on by questionnaire. No identifiable data was collected. All licensed nurses worked in direct patient care were required to participate in two hospital of China.

\subsection{Questionnaire for Fall Prevention Knowledge Development}

The questionnaire consisted of 17 items which used closed-ended responses to collect quantitative data, which has been evaluated for reliability and validity [15]. The questionnaire consisted of 17 items which used closed-ended responses to collect quantitative data. The Likert scale was chosen to provide response options for closed-ended items including the familiarity of strongly unfamiliar, unfamiliar, know a little bit, familiar and strongly familiar which were classified as 1 point, 2 point, 3 point, 4 point and 5 point, respectively.

\subsection{Data Collection Procedure}

Packets for the survey were distributed by nurse managers at the participating agencies and placed it in the locked boxes which were located in each unit nurse station. Each packet contained a cover letter, a demographic information and a fall prevention knowledge questionnaire. There was a poster displayed in the unit nurse station asking for participation. The nurses were instructed via the cover letter to complete the survey on their own time and return to the locked box after finishing. The boxes were emptied every week for two weeks by the first author.

\subsection{Basic Principles and Advantages of Lasso Logistic Regression Model Lasso Logistic}

In 1996, Lasso method was proposed which was characterized by variable selection and complexity adjustment while fitting the generalized linear model [16]. Lasso regression can be modeled regardless of the nature of the target dependent variable. The basic idea of Lasso regression is to minimize the sum of the squared residuals under the condition that the sum of the absolute values of a regression coefficient is less than a constant, so that the coefficient should be zero to zero, and the corresponding variables are deleted to realize the selection of variables. The Logistic regression model is established by selecting variables based on Lasso method.

\subsection{Statistical Analysis}

All quantitative data was managed using Epi Data 3.1 and statistical analysis was managed by R 3.6.2. The least absolute shrinkage and selection operator (LASSO) regression analysis was applied to the relationship between nurses' different care units and their knowledge level of fall prevention. When selecting the optimal Lambda value, relating items of fall prevention were included to analysis and screened by the Logistic regression model, and the nomogram was further constructed. All statistical tests were considered significant with a probability value of less than $0.01(\mathrm{p}<0.01)$.

\section{Results}

\subsection{Sample Description}

A total of 1600 questionnaires were distributed in two acute care hospitals. 1530 recycling questionnaires were collected and there were 1442 valid questionnaires including 1028 questionnaires in general care units and 414 questionnaires in special care units. Questionnaire-reclaiming efficiency was $94.25 \%$. The participants' demographic information was presented in Table 1. 
Table 1. Nurses information data in general care units and special care units of two acute hospitals.

\begin{tabular}{llll}
\hline Items & General care units $(\mathbf{n}, \mathbf{\%})$ & Special care units $(\mathbf{n}, \mathbf{\%})$ & Total $(\mathbf{n}, \mathbf{\%})$ \\
\hline Experience of years & & & \\
$<5$ years & $409(28.36)$ & $151(10.47)$ & $560(38.83)$ \\
$5-9$ years & $288(19.97)$ & $91(6.31)$ & $379(26.28)$ \\
$10-20$ years & $197(13.66)$ & $59(4.09)$ & $256(17.75)$ \\
$>20$ years & $134(9.29)$ & $113(7.84)$ & $247(17.13)$ \\
Education background & & & $573(39.74)$ \\
bachelor Below & $420(29.13)$ & $153(10.61)$ & $869(60.26)$ \\
Bachelor or above & $608(42.16)$ & $261(18.10)$ & $1442(100.00)$ \\
Total & $1028(71.29)$ & $414(28.71)$ & \\
\hline
\end{tabular}

\subsection{The Analysis of Fall Prevention Knowledge Level of Nurses Working on Special Care Units and General Care Units}

Table 2. Comparing the levels of fall prevention knowledge of nurses working on general care units and special care units in 2 acute care hospitals.

\begin{tabular}{|c|c|c|c|}
\hline Items & Questionnaire items' description & Assignment meaning & value \\
\hline 1 & Do you know the definition of a fall? & $0=$ special unit, $1=$ general unit & -0.362 \\
\hline 2 & Are you familiar with the fall prevention assessment tool currently used? & $0=$ special unit, $1=$ general unit & 0.271 \\
\hline 3 & Do you know what people are at high risk of falls? & $0=$ special unit, $1=$ general unit & NA \\
\hline 4 & Do you know when the risk factors of falls should be assessed? & $0=$ special unit, $1=$ general unit & 0.0724 \\
\hline 5 & Do you know what are the risk factors of falls in the environment? & $0=$ special unit, $1=$ general unit & NA \\
\hline 6 & Are you familiar with the assessment of patient's balance and activity? & $0=$ special unit, $1=$ general unit & 0.250 \\
\hline 7 & Do you know any medication that easily causes patients to fall? & $0=$ special unit, $1=$ general unit & 0.176 \\
\hline 8 & Do you know any disease that easily causes patients to fall? & $0=$ special unit, $1=$ general unit & -0.279 \\
\hline 9 & Are you familiar with the assessment of patient's foot problems? & $0=$ special unit, $1=$ general unit & -0.228 \\
\hline 10 & Are you familiar with the assessment of patient's vision? & $0=$ special unit, $1=$ general unit & -0.147 \\
\hline 11 & Are you familiar with the assessment of patients' vertigo and vestibular dysfunction? & $0=$ special unit, $1=$ general unit & NA \\
\hline 12 & Are you familiar with the assessments of the patient's clothing and footwear suitability? & $0=$ special unit, $1=$ general unit & 0.253 \\
\hline 13 & Are you familiar with the assessment of cognitive changes in patient? & $0=$ special unit, $1=$ general unit & 0.025 \\
\hline 14 & Are you familiar with the intervention of fall prevention? & $0=$ special unit, $1=$ general unit & 0.115 \\
\hline 15 & Do you know how to deal with patients after falls? & $0=$ special unit, $1=$ general unit & NA \\
\hline 16 & Are you familiar with the reporting system of falls? & $0=$ special unit, $1=$ general unit & NA \\
\hline 17 & Are you familiar with the reporting procedure of falls? & $0=$ special unit, $1=$ general unit & 0.486 \\
\hline
\end{tabular}

There were total of 17 fall prevention knowledge items in this questionnaire. According to general care units and special care units' nurses working condition, Lasso regression analysis was carried out on the level of nurses' fall prevention knowledge in order to investigate the items of fall prevention knowledge relating to the influence of nurses' care units. Using the $\mathrm{R}$ software package glmnet, 12 items were examined after 10-fold cross validation when Lambda parameter was selected as the minimum value, i.e. Lambda. minequaled to 0.00483 . The meaning of variable assignment was presented in table 2 . The results were obtained by selecting the Lasso regression variable that 12 items screened out included: the definition of fall $(-0.362)$, fall prevention assessment tool currently used (0.271), timing of assessment of the risk factors of falls (0.0724), assessment of patient's balance and activity (0.250), medications that easily cause patients to fall $(0.176)$, diseases that easily cause patients to fall $(-0.279)$, assessment of patient's foot problems $(-0.228)$, assessment of patient's vision (-0.147), assessments of the patient's clothing and footwear suitability $(0.253)$, assessment of cognitive changes in patient (0.025), intervention of fall prevention (0.115) and reporting process of falls (0.486).
Two dashed lines in Figure 1A indicated lambda. min and lambda. 1 respectively. Lambda. min is the lambda value that the mean value of the minimum target parameter which is obtained among all lambda values. Lambda. 1se is the lambda. min value of the simplest model obtained in a variance range of lambda to add a standard error. The optimal lambda. 1se value is 0.01224 and the entries into the model are items 1,2 , $4,6,7,8,9,10,12,13,14,17$ in this study. The vertical coordinate represents the target parameter, the lower coordinate represents the $\log (\lambda)$, and the upper abscissa represents the number of non-zero coefficient variables in the model. For each value, a confidence interval of the target parameter can be obtained by taking the average value of the target parameter shown in the red point.

The Area Under Curve (AUC) was plotted by Lasso analysis and prediction probability which is shown in Figure 1A. Each curve in Figure 1B represents the trajectory of the coefficients of each entry. The $y$-coordinate is the value of the coefficient, the lower $\mathrm{x}$-coordinate is $\log (\lambda)$, and the upper $\mathrm{x}$-coordinate is the number of non-zero coefficients in the model. With the lambda value increasing, the model becomes more compressing, the fewer items enter the model, and the 
ability of the model to select major items becomes stronger.
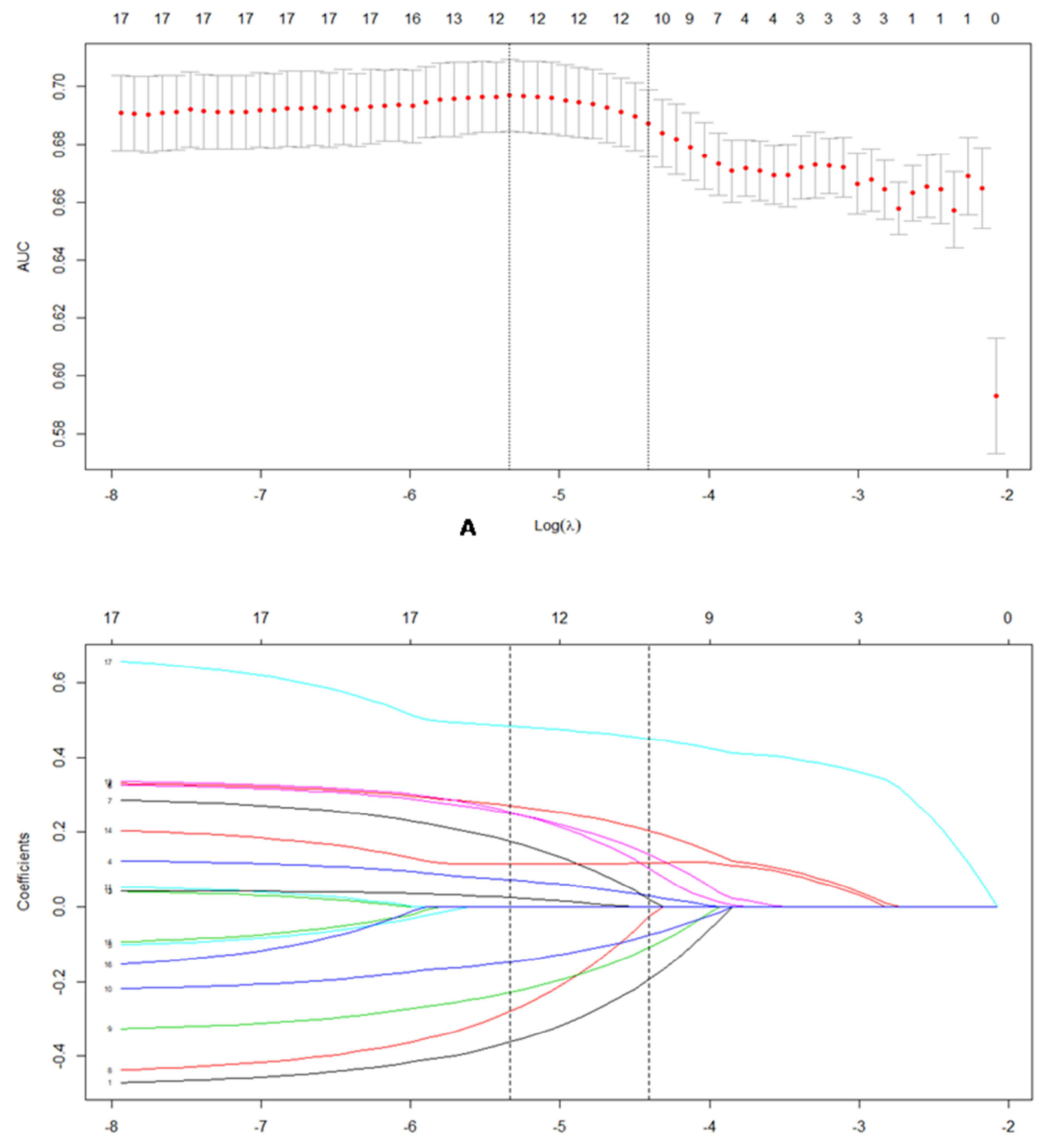

B Log Lambda

Figure 1. Texture feature selection using the least absolute shrinkage and selection operator (Lasso) binary logistic regression model. (A) Tuning parameter selection in the model used 10-fold cross-validation via minimum criteria. The area under the receiver operating characteristic (AUC) curve was plotted versus. Dotted vertical lines were drawn at the optimal values by using the minimum criteria and the 1 standard error of the minimum criteria (the 1 -se. criteria). A $\lambda$ value of 0.01224, with $\log (\lambda),-4.40299$ was chosen (1-secriteria) according to 10-fold cross-validation. (B) LASSO coefficient profiles of the 17 texture features. A coefficient profile plot was produced against the log ( $\lambda$ ) sequence. Vertical line was drawn at the value selected using 10-fold cross-validation, where optimal resulted in 12 nonzero coefficients.

For verification, the Lasso Logistic regression model analysis was performed to confirm a total of 7 items with statistical significance including the definition of a fall $(\mathrm{P}=0.0001)$, fall prevention assessment tool currently used $(\mathrm{p}=0.0053)$, assessment of patient's balance and activity $(p=0.0020)$, diseases that easily cause patients to fall $(\mathrm{p}=0.0021)$, assessment of patient's foot problems ( $\mathrm{p}=0.0068)$, assessments of the patient's clothing and footwear suitability $(p=0.0011)$ and reporting process of falls $(p<0.0001)$.

In this study, the Logistic regression model was established to review the results of Lasso variable selection, indicating that its fitting and prediction effects were appropriate. The 
results were shown in table 3.

Table 3. Lambda. Ise Logistic regression analysis of the relationship between different care units of nurses and their knowledge level of fall prevention.

\begin{tabular}{|c|c|c|c|c|}
\hline Items & Coef. & S.E. & Walds & P Value \\
\hline 1. Do you know the definition of a fall? & -0.4855 & 0.1267 & -3.83 & $0.0001 *$ \\
\hline 2. Are you familiar with the fall prevention assessment tool currently used? & 0.3203 & 0.1148 & 2.79 & $0.0053 *$ \\
\hline 4. Do you know when the risk factors of falls should be assessed? & 0.1033 & 0.1243 & 0.83 & 0.4059 \\
\hline 6. Are you familiar with the assessment of patient's balance and activity? & 0.3299 & 0.1069 & 3.09 & $0.0020^{*}$ \\
\hline 7. Do you know any medication that easily cause patients to fall? & 0.2863 & 0.1275 & 2.25 & 0.0247 \\
\hline 8. Do you know any disease that easily cause patients to fall? & -0.4627 & 0.1505 & -3.07 & $0.0021 *$ \\
\hline 9. Are you familiar with the assessment of patient's foot problems? & -0.3181 & 0.1176 & -2.71 & $0.0068^{*}$ \\
\hline 10. Are you familiar with the assessment of patient's vision? & -0.1993 & 0.0998 & -2.00 & 0.0458 \\
\hline 12. Are you familiar with the assessments of the patient's clothing and footwear suitability? & 0.3591 & 0.1096 & 3.28 & $0.0011 *$ \\
\hline 13. Are you familiar with the assessment of cognitive changes in patient? & 0.0489 & 0.1154 & 0.42 & 0.6719 \\
\hline 14. Are you familiar with the intervention of fall prevention? & 0.1176 & 0.1402 & 0.84 & 0.4016 \\
\hline 17. Are you familiar with the reporting process of falls? & 0.5165 & 0.1039 & 4.97 & $<0.0001 *$ \\
\hline
\end{tabular}

Notes. The items marked * mean statistical differences.

\section{Discussion}

1442 clinical nurses from 2 acute care hospitals in Guangzhou participated in our study. They were divided into two groups: 1028 nurses in general care units including medical, surgical, obstetrics and pediatrics; and 414 nurses in special care units including emergency department, ICU and operating room. The knowledge levels of the 7 fall prevention items in two groups of nurses were different by using the Lasso regression model.

On one hand, in this study we found that the knowledge level of nurses in special care units was higher than that of nurses in general care units in terms of the definition of a fall, diseases that easily cause patients to fall and assessment of patient's foot problems. Possible reasons for this result are as follows: special care units such as ICU, operating room and emergency department have higher requirements on nurses' core competence and professional quality [17-19]. Nurses in these units are equipped with high education or rich working experience, and their nursing theoretical foundation more solid than that of nurses in general care units. It demonstrated that the proportion of ICU nurses with bachelor degree or above was $66 \%$ in $\mathrm{Li} \mathrm{W}$. study on the work status of ICU nurses and the analysis of influencing factors [20]. In this study, we found that the proportion of nurses with bachelor's degree or above in special care units was about $4 \%$ higher than that in general care units, which suggested that the Department of Quality and Patient Safety in medical institution should strengthen the training of nurses about the theoretical knowledge relating to fall prevention in general care units.

On the other hand, in this study we found out that the knowledge level of nurses in special care units was lower than that of nurses in general care units in terms of fall prevention assessment tool, assessment of patient's balance and activity, assessments of the patient's clothing and footwear suitability and reporting process of falls. In terms of fall risk assessment tools, special care units of Emergency Department, ICU and Operating Room have no appropriate fall risk assessment tools to be used, and most of nurses use self-made fall risk assessment tools or make oral assessments of patients [21, 22,
10]. One study showed that the use of the correct fall risk assessment tools to screen the high-risk groups of falls, early identification of the risk factors of falls and nursing intervention could reduce the incidence of falls in hospitalized patients from 0.3 to $0.1 \%$ [ [23]. In the recent years, there were a large number of fall risk assessment tools. The fall risk assessment tools applied to inpatients in general care units is more common in China, while there is a lack of fall assessment tool with specialty characteristics for special care units [24]. Accordingly, we need to conduct scientific research on fall assessment tools and develop fall risk assessment tools that are suitable for special care units. Moreover, Emergency Department, Operating room, and ICU are the main wards for critical care and treatment in the hospital that their primary objectives of care are first aid and maintenance of patients' vital signs and basic physiological needs. Nurses in these special care units pay less attention and practice rarely on patients' fall prevention such as the lack of assessment of patients' balance and mobility, and the assessment of the appropriateness of clothing and shoes, etc. [25], and they are not familiar with the fall reporting process.

\section{Conclusion}

In this study, we can conclude that nurses in special care units had high level theoretical knowledge of fall prevention than nurses in general care units, while they had lower level practical knowledge of fall prevention. It suggested that Quality \& Patient Safety Department in medical institution should make new plans to prevent patients from falling and ensure patient safety. In this study we only studied the influence of nurses working on different care units with fall prevention knowledge. Further investigation and discussion on the educational level and seniority of nurses relate to the knowledge of fall prevention will be conducted.

\section{Acknowledgements}

This study was supported by grants from Guangdong Medical Science and Technology Research Foundation (Grant No. A2020362, A2019120). 
We would like to thank all of the participants who took part in this study and Professor Elizabeth Roe and Professor Sally Decker who provided guidance to us and help at Saginaw Valley State University in Michigan State, USA.

\section{References}

[1] World Health Organization (2018). Falls (Fact sheet). Retrieved from http://www.who.int/mediacentre/factsheets/fs34/en/

[2] Zhang, Y. and Chen W. (2008). Overview and progress of the study on the fall of old age. Chinese Journal of Gerontology, 28 (5), 929-931.

[3] Nurmi I. and Luthje P. (2002). Incidence and costs of falls and fall injuries among Elderly in Institutional Care. Scand J Prim Health Care, 20 (2): 118-122.

[4] Lori, L., and Julie, M. (2010). The physical environments as a fall risk factor in older adults: systematic review and meta-analysis of cross-sectional and cohort studies. Australian Occupational Therapy Journal, 57, 51-64.

[5] Huang Q., Lu S. P., Xu Y. N., et al. (2017). Construction of Evaluation Index System of Falls in Elderly Hospitalized Patients Based on Nursing-sensitive Outcomes. Chinese Journal of Nursing, 24 (22), 63-66.

[6] Li M., Liu W. P., Bai X. et al. (2019). Analysis of fall related factors in hospitalized elderly patients in internal medicine system [J]. Nursing research, 33 (08): 1447-1449.

[7] Lu Y. N. (2008). Prevention and countermeasures of fall risk in surgical inpatients. Journal of Traditional Chinese Medicine Management, 26 (20): 163-165.

[8] Fu S. H. (2015). Application of systematic management mode in the prevention of falls in gynecological patients. Journal of Traditional Chinese Medicine Management, 23 (03): 67-68.

[9] Huang X. H. and Li W. T. (2017). Application of medical sharing platform in pediatric nursing risk assessment. Journal of Nursing Science, 32 (09): 18-19.

[10] Li C. P. (2015). Implementation and effect observation of fall prevention measures in emergency observation room. World Latest Medical Information Digest, 15 (A4): $260+275$.

[11] Qu S. (2019). Observation on the application effect of medical integration model in the continuous improvement of nursing quality in the operating room. Chinese Medical Guidelines, 17 (33): 318-319.

[12] Chen H., Shi Z. X., Zhang W. Y., et al. (2017). Case characteristics and countermeasures of elderly patients falling and falling into bed in cardiac intensive care unit. Continuing Medical Education, 31 (04): 111-113.
[13] Zhu C. Q., Song Y. and Yang S. W. (2010). Investigation on nurses' cognition of falls in hospitalized elderly patients. Jilin Medical Science, 31 (16): 2456-2457.

[14] Yan W., Chen Q., Zhang X. M., et al. (2019). Survey on knowledge and attitude of nurses in intervention guidelines for falls in the elderly in Sichuan province in China. Nursing research, 33 (12): 2126-2129.

[15] Gong X. and Chen W. j. (2014). Investigation on knowledge, attitudes and behaviors of clinical nurses in prevention of inpatients' falls and its countermeasures. Chinese Nursing Research, 28 (4c), 1428-1430.

[16] Robert Tibshirani. (1996). Regression shrinkage and selection via the Lasso. Journal of the Royal Statistical Society, Series B, 58 (1): $267-288$.

[17] Zhang X., Chen M. R., Xu B. F., et al. (2019). Study on the status quo and influencing factors of core competency of ICU nurses in 12 hospitals in Fujian province. Chinese Nursing Management, 19 (03): 404-409.

[18] Chen X. J. and Zhao J. H. (2008). Research progress on core competence of emergency department nurses. International Journal of Nursing, 37 (04): 444-446 + 473.

[19] Mu Li, Wang Z., Jiang L. P., et al. (2019). Investigation and analysis of the core competence of professional nurses in operating room and its influencing factors. Chinese Journal of Medical Education, 39 (03): 231-235.

[20] Li W., Cao J., Wang Y. Y., et al. (2020). Analysis on the status quo and influencing factors of ICU nurse work embedding. Chinese Nursing Management, (02): 229-233.

[21] Liu Q., Lei Y. H., Liu J. L., et al. (2008). Applicability of Chinese Morse fall assessment scale in ICU patients. Bachu Medicine, 1 (01): 102-106.

[22] Qian H. J., Zhang L. Q. Chen Y. F., et al. (2010). Application of fall risk factor assessment table in operating room nursing. Chinese Journal of Practical Nursing, 26 (21): 58-59.

[23] Mei X. Q., Ji X. M., Zhang C., et al. (2016). Effect observation of reducing the incidence rate of fall in inpatients by the supply management circle. Chinese Health Education, 32 (09): 849-851.

[24] Zhou X. M. and Feng X. (2008). Research progress of fall risk assessment tools. Journal of nursing, 33 (21): 109-112.

[25] Xue H. Y. and Yu Z. Y. (2019). Research progress on fall risk factors in emergency room patients. Chinese Medical Management Science, 9 (02): 55-60. 